\title{
Indoleamine 2,3-dioxygenase, by degrading L-tryptophan, enhances carnitine palmitoyltransferase I activity and fatty acid oxidation, and exerts fatty acid-dependent effects in human alloreactive $\mathrm{CD4}^{+} \mathrm{T}$-cells
}

\author{
THEODOROS ELEFTHERIADIS ${ }^{1}$, GEORGIOS PISSAS ${ }^{1}$, MARIA SOUNIDAKI $^{1}$, KONSTANTINA TSOGKA $^{1}$, \\ NIKOLAOS ANTONIADIS ${ }^{2}$, GEORGIA ANTONIADI ${ }^{1}$, VASSILIOS LIAKOPOULOS ${ }^{1}$ and IOANNIS STEFANIDIS ${ }^{1}$ \\ ${ }^{1}$ Department of Nephrology, Medical School, University of Thessaly, Larissa; \\ ${ }^{2}$ Organ Transplant Unit, Medical School, Aristotle University of Thessaloniki, Thessaloniki, Greece
}

Received May 14, 2016; Accepted September 9, 2016

DOI: $10.3892 /$ ijmm.2016.2750

\begin{abstract}
Indoleamine 2,3-dioxygenase (IDO) is expressed in antigen-presenting cells and by degrading L-tryptophan along the kynurenine pathway suppresses $\mathrm{CD} 4^{+} \mathrm{T}$-cell proliferation, induces apoptosis and promotes differentiation towards a regulatory as opposed to an effector phenotype. Recent findings revealed that the above effects may be mediated through alterations in T-cell metabolism. In this study, the effect of IDO on fatty acid $\beta$-oxidation in $\mathrm{CD}^{+}{ }^{+} \mathrm{T}$-cells was evaluated in human mixed lymphocyte reactions (MLRs) using the IDO inhibitor, 1-DL-methyl-tryptophan. Protein analysis of $\mathrm{CD}^{+} \mathrm{T}$-cells isolated from the MLR showed that L-tryptophan degradation acts by activating the general control non-derepressible 2 kinase and aryl-hydrocarbon receptor in T-cells. In the absence of IDO inhibition, fatty acid oxidation increased along with increased activity of carnitine palmitoyltransferase I (CPT1), the latter due to the increased expression of CPT1 isoenzymes and alterations in acetyl-CoA carboxylase 2, the enzyme that controls CPT1 activity. Increased fatty acid oxidation due to the action of IDO was accompanied by an increased expression of forkhead box P3 (FoxP3) and a decreased expression of related orphan receptor $\gamma \mathrm{t}(\mathrm{ROR} \gamma \mathrm{t})$, the signature transcription factors of regulatory T-cells and T helper 17 cells, respectively. However, in MLR and in the presence of fatty acid in the culture medium, IDO did not inhibit proliferation. Additionally, fatty acid protected the $\mathrm{CD} 4^{+} \mathrm{T}$-cells against apoptosis. Thus, IDO, by degrading L-tryptophan, enhances CPT1 activity and fatty acid oxidation, and exerts fatty acid-dependent effects in human alloreactive $\mathrm{CD} 4^{+} \mathrm{T}$-cells.
\end{abstract}

Correspondence to: Professor Theodoros Eleftheriadis, Department of Nephrology, Medical School, University of Thessaly, Neo Ktirio, Mezourlo Hill, 41110 Larissa, Greece

E-mail: teleftheriadis@yahoo.com

Key words: indoleamine 2,3-dioxygenase, fatty acid, carnitine palmitoyltransferase I, $\mathrm{CD} 4^{+} \mathrm{T}$-cells, proliferation, differentiation

\section{Introduction}

Indoleamine 2,3-dioxygenase (IDO) is expressed in antigen-presenting cells (APCs) and has an immunoregulatory role in various models of autoimmunity and allotransplantation (1-6). In addition to APCs, IDO is expressed in trophoblast cells contributing to successful semi-allogenic pregnancy $(7,8)$, while its expression in certain cancer cells has been incriminated for escape of cancer from immunosurveillance (9). In hemodialysis patients, who are characterized by impaired adaptive immunity, increased serum IDO levels have been associated with decreased T-cell count, as well as failure to respond to a vaccine with a T-cell dependent antigen $(10,11)$.

By degrading L-tryptophan along the kynurenine pathway, IDO alters the local microenvironment in a manner that suppresses T-cell function. More precisely, L-tryptophan depletion activates general control non-derepressible 2 kinase $(\mathrm{GCN} 2 \mathrm{~K})$, which phosphorylates eukaryotic initiation factor $2 \alpha(\mathrm{eIF} 2 \alpha)$, altering the translational program of T-cells (12-16). The effect of L-tryptophan depletion on the other amino acid sensing system, the mammalian target of rapamycin complex 1 (mTORC1), has also been investigated although with contradictory results (12-17). Kynurenine, a product of L-tryptophan degradation by IDO, by activating the aryl-hydrocarbon receptor (AhR) is involved in the immunosuppressive action of this enzyme favoring $\mathrm{CD}^{+} \mathrm{T}$-cell differentiation towards regulatory T-cells (Treg) $(18,19)$.

Considering the above described pathways as a starting point, the presence of IDO in APCs leads to decreased proliferation and increased apoptosis, and promotes the differentiation of $\mathrm{CD}^{+} \mathrm{T}$-cells towards a regulatory instead of effector (Teff) phenotype $(20,21)$. While many intermediate events remain to be elucidated, recent research indicates that IDO may exert these effects by affecting the metabolism of $\mathrm{CD}^{+} \mathrm{T}$-cells. Indeed, IDO suppresses aerobic glycolysis and glutaminolysis in human alloreactive $\mathrm{CD} 4^{+} \mathrm{T}$-cells by affecting the expression of glucose transporter 1 and various glycolytic and glutaminolytic enzymes (14-16). It also downregulates key enzymes involved in fatty acid synthesis (17). The above-mentioned 
metabolic pathways are prerequisites for rapid T-cell proliferation following T-cell receptor stimulation, as well as for differentiation towards effector cell lineages instead of Treg. Following T-cell activation, T-cells reprogram their metabolic pathways from pyruvate via the Krebs cycle to the glycolytic and glutaminolytic pathways in order to fulfill the bioenergetic and biosynthetic demands of proliferation (22-24). In parallel, fatty acid synthesis is upregulated during the activation of $\mathrm{CD} 4^{+}$ T-cells enhancing their proliferation and promoting their differentiation into T helper 17 cells (Th17) instead of Tregs (25).

Another metabolic pathway that plays a significant role in the $\mathrm{CD} 4^{+} \mathrm{T}$-cell response and differentiation is fatty acid $\beta$-oxidation. More precisely, Tregs are dependent on fatty acid oxidation for its differentiation, whereas, Teff populations on aerobic glycolysis (26). The effect of IDO on fatty acid oxidation in $\mathrm{CD}^{+} \mathrm{T}$-cells remains to be investigated, and constitutes the aim of the present study.

For the purposes of the present study, two-way mixed lymphocyte reaction (MLR) was used as a model of alloreactivity (27), along with the specific IDO inhibitor, 1-DL- methyl-tryptophan (1MT) $(4,7)$. In order to evaluate fatty acid oxidation, cells were cultured in a medium containing oleate. The effect of IDO on carnitine palmitoyltransferase I (CPT1), the tightly regulated enzyme that controls the entry of fatty acids into the mitochondria for oxidation $(28,29)$, was assessed as well. Treatment of $\mathrm{CD} 4^{+} \mathrm{T}$-cells with the CPT1 inhibitor, etomoxir, has been shown to abrogate differentiation into Tregs (26). The effects of IDO on the end-points of $\mathrm{CD} 4^{+} \mathrm{T}$-cell function, proliferation, apoptosis and differentiation were also evaluated.

\section{Materials and methods}

Subjects. Blood samples were collected from 5 healthy volunteers ( 3 males and 2 females, $37 \pm 7$ years of age). Informed consent was obtained from each individual enrolled in the study and the Ethics Committee of the University Hospital of Larissa (Larissa, Greece) approved the study protocol.

Cell culture conditions. Peripheral blood mononuclear cells (PBMCs) were isolated from whole blood by the Ficoll-Hypaque density gradient centrifugation (Histopaque 1077; Sigma-Aldrich, St. Louis, MO, USA) and counted using an optical microscope (Axiovert $40 \mathrm{C}$; Carl Zeiss AG, Oberkochen, Germany) on a Neubauer plaque. Cell viability was assessed by trypan blue assay (Sigma-Aldrich). Cell cultures were performed in RPMI-1640 medium with L-glutamine, $10 \mathrm{mM}$ 4-(2-hydroxyethyl)-1-piperazineethanesulfonic acid (HEPES) and supplemented with $10 \%$ fetal bovine serum and antibiotic-antimycotic solution (both from Sigma-Aldrich). The cultures were performed at $37^{\circ} \mathrm{C}$ in a humidified atmosphere containing $5 \% \mathrm{CO}_{2}$.

Ten MLRs were performed in the presence or absence of the IDO inhibitor 1MT (Sigma-Aldrich) at a concentration of $100 \mu \mathrm{M}$. The above concentration was chosen according to previous experiments that revealed efficacy without toxicity (13-16). Unless otherwise stated, in all the experiments, oleate (Sigma-Aldrich) at a final concentration of $1 \mathrm{mM}$ was added from the beginning of the MLRs.

To determine cell proliferation, 10 MLRs were performed in 96-well plates for 7 days. Peripheral blood mononuclear cells from each member of the MLR couple were $5 \times 10^{4}$, measuring to $1 \times 10^{5}$ PBMCs in total in each well. Cultures of $1 \times 10^{5} /$ well resting PMBCs from each member of the MLR couple were used as controls.

To assess various components in the supernatant, as well as the expression of certain proteins and CPT1 activity in $\mathrm{CD}^{+} \mathrm{T}$-cells, 10 MLRs were performed in 12-well plates for 7 days. The number of PBMCs for each member of the MLR couple was $5 \times 10^{5}$, reaching a total of $1 \times 10^{6}$ PBMCs in each well. At the end of the 7-day period supernatants were collected and stored at $-80^{\circ} \mathrm{C}$, whereas $\mathrm{CD} 4^{+} \mathrm{T}$-cells were isolated from the MLRs by negative selection using the $\mathrm{CD} 4^{+} \mathrm{T}$-cell isolation kit, human (Miltenyi Biotec GmbH, Bergisch Gladbach, Germany).

Cell proliferation in two-way mixed lymphocyte reactions. Cell proliferation enzyme-linked immunosorbent assay (ELISA) (Roche Diagnostics, Indianapolis, IN, USA), based on bromodeoxyuridine (BrdU) labeling and immunoenzymatic detection, was used to examine cell proliferation. The proliferation index was calculated as the ratio of the optical density (OD) derived from each MLR to the mean of the ODs derived from the control resting PBMC cultures of the two subjects that constituted the specific MLR. These experiments were performed in triplicate and the results refer to the mean of the three measurements.

\section{L-tryptophan and oleate consumption in MLRs. L-tryptophan} consumption was assessed by measuring its concentration in the supernatants of MLRs by means of ELISA (BlueGene Biotech, Shanghai, China). The sensitivity of the above ELISA kit is $1 \mathrm{ng} / \mathrm{ml}$.

Similarly, oleate consumption was assessed by measuring its concentration in the supernatants of MLRs colorimetrically using the Free Fatty Acid Quantification kit (Abcam, Cambridge, UK). The detection limit of the above kit was $2 \mu \mathrm{M}$.

Expression of certain proteins in $C D 4^{+} T$-cells isolated from the MLRs. The expression of certain proteins in $\mathrm{CD} 4^{+} \mathrm{T}$-cells was assessed by western blot analysis. Isolated CD4 ${ }^{+}$ T-cells were counted via optical microscopy on a Neubauer plaque and cell viability was determined by trypan blue assay (Sigma-Aldrich). Equal numbers of T-cells from each MLR were lysed using the T-PER tissue protein extraction reagent (Thermo Fisher Scientific Inc., Rockford, IL, USA) supplemented with protease and phosphatase inhibitors (Sigma-Aldrich and Roche Diagnostics). Protein was quantified using the Bradford assay (Sigma-Aldrich) and $10 \mu \mathrm{g}$ from each sample were used for western blot analysis. The blots were incubated with the primary antibodies for $16 \mathrm{~h}$, followed by the secondary antibody (anti-rabbit IgG, HRP-linked antibody; Cell Signaling Technology, Danvers, MA, USA) incubation for 30 min. In case of reprobing PVDF blots, the previous primary and secondary antibodies were removed using the Restore Western Blot Stripping Buffer (Thermo Fisher Scientific Inc.) according to the manufacturer's protocol. The PVDF blot was then reused and western blot analysis resumed as previously described, using a different primary antibody. Analysis of the western blots was performed using the ImageJ software (National Institute of Health, Bethesda, MD, USA).

The primary antibodies used in western blot analysis were specific for eIF $2 \alpha$ phosphorylated at serine 51 (p-eIF2 $\alpha$; 
Cat. no. 9721) (Cell Signaling Technology), cytochrome P450, family 1, subfamily A, polypeptide 1(CYP1A1; Cat.no. sc-20772) (Santa Cruz Biotechnology,Inc., Dallas, TX, USA), p-70S6 kinase phosphorylated at threonine 389 (p-p70S6K; Cat. no. 9234) (Cell Signaling Technology), CPT1A (Cat. no. 12252S; Cell Signaling Technology), CPT1B (Cat. no. ab134988), CPT1C (Cat. no. ab87498) (both from Abcam), acetyl-CoA carboxylase 2 (ACC2; Cat. no. 8578) (Cell Signaling Technology), ACC2 phosphorylated at serine 221 (p-ACC2; Cat. no. ab109540) (Abcam), activated cleaved at aspartate 175 caspase-3 (Cat. no. 9664), forkhead box P3 (FoxP3; Cat. no. 5298) (both from Cell Signaling Technology), retinoic acid receptor related orphan receptor $\gamma t$ (ROR $\gamma$ t; Cat. no. orb6888) (Biorbyt, Cambridge, UK) and $\beta$-actin (Cat. no. 4967; Cell Signaling Technology).

Carnitine palmitoyltransferase I activity. To determine CPT1 enzyme activity, a non-radioactive method was performed in whole cell lysates according to the method of Bieber and Fiol (30). CD4 ${ }^{+}$T-cell lysates were prepared as described for western blot analysis and the method was based on measurement of the initial release of CoA-SH from palmitoyl CoA specrtrophotometrically using the reagent 5,5'-dithio-bis-(2-nitrobenzoic acid) (DTNB). Briefly, $50 \mu 1$ buffer solution (containing $116 \mathrm{mM}$ Tris, $2.5 \mathrm{mM}$ EDTA, $2 \mathrm{mM}$ DTNB, $0.2 \%$ Triton $\mathrm{X}-100, \mathrm{pH} 8.0$ ) and $50 \mu \mathrm{g}$ protein extract were added to the reaction mixture. After $5 \mathrm{~min}$ preincubation at $28^{\circ} \mathrm{C}$, $50 \mu \mathrm{l}$ of $1 \mathrm{mM}$ palmitoyl-CoA was added and the reaction was initiated with a final addition of $5 \mu \mathrm{l}$ of $1.2 \mathrm{mM}$ L-carnitine solution, followed by an immediate photometric measurement at $412 \mathrm{~nm}$. These reagents were purchased from Sigma-Aldrich.

Statistical analysis. The normality of the evaluated variables was assessed and confirmed by the one-sample Kolmogorov-Smirnov test. For comparison of means the paired-sample t-test or unpaired-sample t-test were used. Results were presented as the means \pm standard deviation (SD). A value of $\mathrm{P}<0.05$ was considered to indicate a statistically significant difference.

The results obtained from the western blot analysis and enzyme activity assay are expressed as optical densities (OD), thus p-values were calculated by comparing the means of OD. Statistical analysis after normalization for the control OD values was avoided to prevent violation of the prerequisite for normal distribution of the compared variables when applying parametric statistical tests. However, for the reader's convenience, in the text and figures the results are noted and depicted after normalization of means for the control group.

\section{Results}

IDO increases L-tryptophan degradation in MLRs, enhances eIF $2 \alpha$ phosphorylation and CYP1A1 expression in MLR-derived $\mathrm{CD}^{+} \mathrm{T}$-cells, but does not affect $\mathrm{p} 70 \mathrm{~S} 6 \mathrm{~K}$ phosphorylation in MLR-derived $C D 4^{+} T$-cells. In MLRs, IDO increased L-tryptophan degradation since its inhibitor 1MT increased L-tryptophan concentration in the supernatants from $2.47 \pm 0.44$ to $6.19 \pm 0.47 \mu \mathrm{g} / \mathrm{ml}$ (p<0.001, paired t-test) (Fig. $1 \mathrm{~A})$.

By degrading L-tryptophan, IDO enhanced the p-eIF2 $\alpha$ level in MLR-derived CD4 ${ }^{+}$T-cells since the treatment of MLRs with
$1 \mathrm{MT}$ altered the p-eIF $2 \alpha$ level by a factor of $0.52 \pm 0.18(\mathrm{p}<0.001$, paired t-test) (Fig. 1B and C). Similarly, in $\mathrm{CD}^{+}{ }^{+} \mathrm{T}$-cells derived from 1MT-treated MLRs, CYP1A1 expression was altered by a factor of $0.51 \pm 0.29(\mathrm{p}<0.001$, paired $\mathrm{t}$-test $)$ indicating that IDO increases CYP1A1 expression (Fig. 1B and D). By contrast, 1MT treatment of the MLRs did not affect the content of p-p70S6K in $\mathrm{CD} 4^{+} \mathrm{T}$-cells, since its level was altered only by a factor of $1.07 \pm 0.16$ ( $\mathrm{p}=0.316$, paired t-test) (Fig. $1 \mathrm{~B}$ and $\mathrm{E})$.

IDO increases fatty acid oxidation in MLRs and CPT1 enzymatic activity in MLR-derived $C D 4^{+} T$-cells. In MLRs, IDO increased fatty acid oxidation since its inhibitor $1 \mathrm{MT}$ increased the oleate concentration in the supernatants from $0.51 \pm 0.05 \mathrm{mM}$ to $0.82 \pm 0.03 \mathrm{mM}$ (p<0.001, paired t-test) (Fig. 2A).

In CD4 ${ }^{+}$T-cells derived from 1MT-treated MLRs CPT1 activity was at the $56.61 \pm 7.32 \%$ of the activity found in cells derived from the control MLRs $(\mathrm{p}<0.001$, paired $\mathrm{t}$-test), indicating that IDO enhanced CPT1 enzymatic activity in CD4 ${ }^{+}$T-cells (Fig. 2B).

IDO increases CPTIA, CPTIB and CPTIC expression in $M L R$-derived $C D 4^{+} T$-cells. Unblocked IDO activity in MLRs increased CPT1A expression in MLR-derived CD4 ${ }^{+}$T-cells since the treatment of MLRs with the IDO inhibitor, 1MT, altered the CPT1A level by a factor of $0.74 \pm 0.05(\mathrm{p}<0.001$, paired t-test) (Fig. 3A and B). This was even more profound in the case of CPT1B, which was altered due to $1 \mathrm{MT}$ by a factor of $0.57 \pm 0.13$ ( $p<0.001$, paired t-test) (Fig. 3A and C), and of CPT1C, which was altered by a factor of $0.44 \pm 0.13(\mathrm{p}<0.001$, paired t-test) (Fig. 3A and D).

IDO decreases ACC2 expression, whereas it increases the level of phosphorylated ACC2 in MLR-derived $C D 4^{+} T$-cells. IDO activity in the MLRs decreased the total ACC2 expression in MLR-derived CD4 ${ }^{+}$T-cells since the treatment of MLRs with the IDO inhibitor, 1MT, led to alterations in the levels of ACC 2 by a factor of $1.24 \pm 0.18(\mathrm{p}=0.001$, paired t-test) (Fig. 4A and B).

The effect of IDO on the level of p-ACC2 was more profound since in the $\mathrm{CD}^{+}{ }^{+} \mathrm{T}$-cells derived from the 1MT-treated MLRs, the level of p-ACC2 was altered by a factor of $0.37 \pm 0.18$ ( $p<0.001$, paired t-test) (Fig. 4A and C). Thus, IDO, by degrading L-tryptophan in the MLRs, increased the content of the inactivated phosphorylated form of ACC2 in CD4 ${ }^{+}$T-cells.

IDO does not affect cell proliferation in MLRs nor activated caspase-3 in MLR-derived $C D 4^{+} T$-cells. Using culture media containing oleate, IDO did not affect cell proliferation in MLRs, since the addition of 1MT did not affect the proliferation index significantly. More precisely, the proliferation index was $4.87 \pm 0.27$ in the untreated MLRs and $4.75 \pm 0.34$ in the 1MT-treated MLRs ( $\mathrm{p}=0.313$, paired t-test) (Fig. 5A).

Similarly, in the presence of oleate, IDO did not affect the content of activated caspase- 3 in $\mathrm{CD}^{+}{ }^{+} \mathrm{T}$-cells, which is the terminal caspase of the apoptotic pathways. Compared to the activated caspase- 3 level in $\mathrm{CD}^{+}{ }^{+} \mathrm{T}$-cells derived from the control MLRs, its level did show a negligible variation only by a factor of $0.98 \pm 0.11$ in $\mathrm{CD}^{+}{ }^{+} \mathrm{T}$-cells derived from $1 \mathrm{MT}$-treated MLRs ( $p=0.523$, paired t-test) (Fig. 5B and C). 

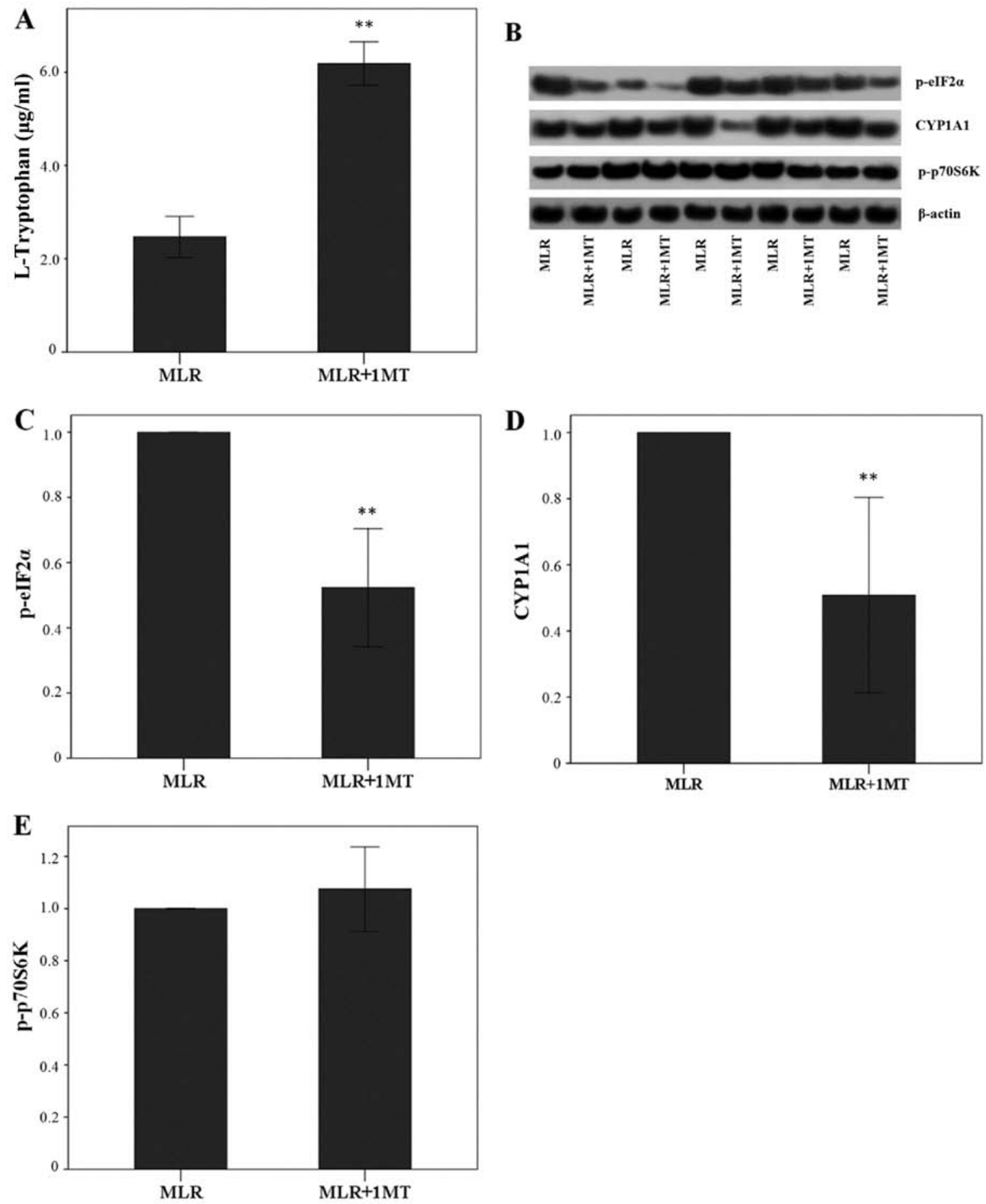

Figure 1. Effect of indoleamine 2,3-dioxygenase (IDO) on L-tryptophan degradation in mixed lymphocyte reactions (MLRs) and on the expression of p-eukaryotic initiation factor $2 \alpha$ (eIF2 $\alpha$ ), cytochrome P450, family 1, subfamily A, polypeptide 1 (CYP1A1) and p-p70S6K in MLR-derived CD4+ T-cells. Ten MLRs were performed in the presence or absence of the IDO inhibitor, 1-DL-methyl-tryptophan (1MT); the supernatants were collected and CD4 ${ }^{+}$T-cells were isolated for the evaluation of the expression of certain proteins. (A) IDO increased L-tryptophan degradation in MLRs. (B) The results of western blot analysis of 5 of the 10 performed experiments are shown. IDO enhanced the level of (C) p-eIF2 $\alpha$ and (D) CYP1A1 level, while it did not affect the level of (E) p-p70S6K level in MLR-derived T-cells. Error bars represent the means \pm SD; * ${ }^{*}<0.001$ compared to MLR-devided cells.

IDO, particularly in the presence of oleate, induces FoxP3 expression, but suppresses ROR $\gamma$ texpression in MLR-derived $C D 4^{+} T$-cells. Unblocked IDO activity in MLRs increased FoxP3 expression in MLR-derived $\mathrm{CD}^{+}{ }^{+} \mathrm{T}$-cells as the treatment of MLRs with the IDO inhibitor, 1MT, altered the FoxP3 level by a factor of $0.33 \pm 0.21(\mathrm{p}<0.001$, paired t-test) (Fig. 6A and B).
The opposite was observed with the expression of ROR $\gamma \mathrm{t}$. Treatment of the MLRs with the IDO inhibitor, 1MT, induced a significant increase in ROR $\gamma$ t levels by a factor of $2.24 \pm 0.41$ ( $\mathrm{p}<0.001$, paired t-test) (Fig. 6A and C). Thus, by degrading L-tryptophan, IDO decreased ROR $\gamma$ t expression in CD $4^{+}$T-cells.

In the absence of oleate, treatment of the MLRs with 1MT also resulted in a decrease in FoxP3 expression in $\mathrm{CD} 4{ }^{+} \mathrm{T}$-cells 

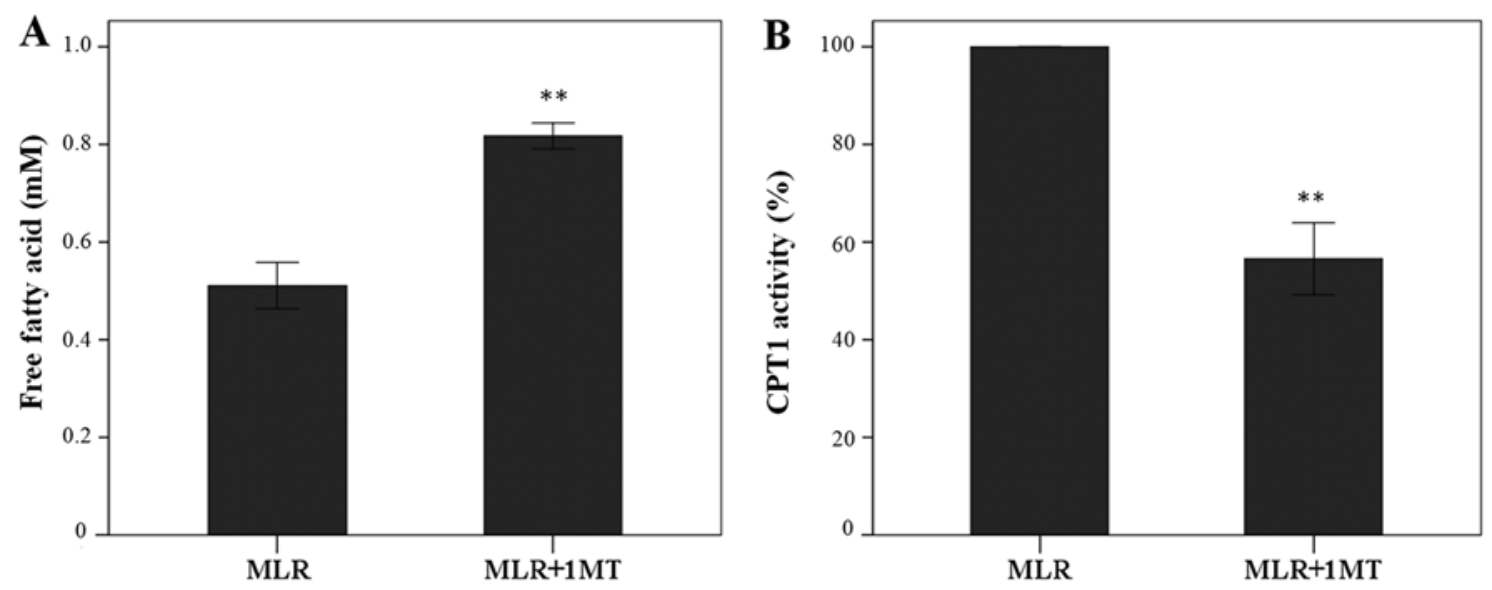

Figure 2. Effect of indoleamine 2,3-dioxygenase (IDO) on fatty acid oxidation in mixed lymphocyte reactions (MLRs) and on carnitine palmitoyltransferase I (CPT1) activity in MLR-derived CD4+ T-cells. Ten MLRs were performed in the presence or absence of the IDO inhibitor, 1-DL-methyl-tryptophan (1MT), the supernatants were collected and CD4 $4^{+}$T-cells were isolated to evaluate CPT1 enzymatic activity. (A) IDO increased fatty acid oxidation in MLRs. (B) IDO enhanced CPT1 enzymatic activity in MLR-derived CD4 ${ }^{+} \mathrm{T}$ cells. Error bars represent the means $\pm \mathrm{SD} ;{ }^{* *} \mathrm{p}<0.001$ compared to MLR-devided cells.

A

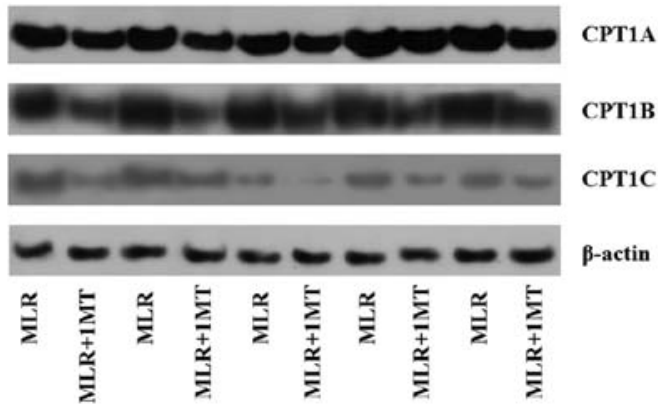

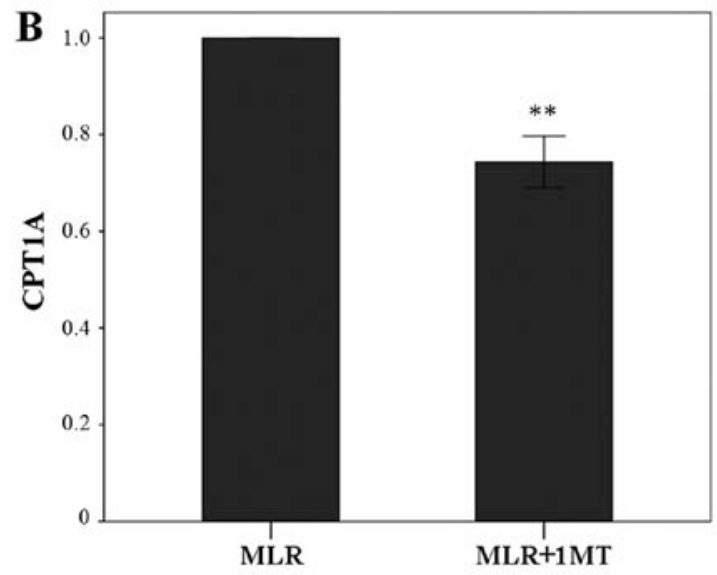

D

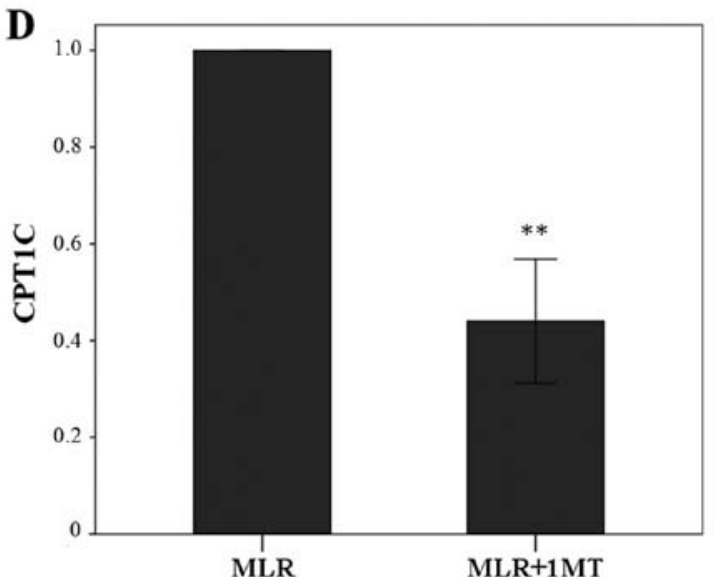

Figure 3. The effect of indoleamine 2,3-dioxygenase (IDO) on carnitine palmitoyltransferase IA (CPT1A), CPT1B and CPT1C expression in MLR-derived $\mathrm{CD}^{+}{ }^{+}$T-cells. Ten mixed lymphocyte reactions (MLRs) were performed in the presence or absence of the IDO inhibitor 1-DL-methyl-tryptophan (1MT), and $\mathrm{CD}^{+} \mathrm{T}$-cells were isolated for evaluating the expression of CPT1 isoenzymes. (A) Western blot analysis results of 5 of the 10 performed experiments are shown. IDO increased the expression of the isoenzymes (B) CPT1A, (C) CPT1B and (D) CPT1C in MLR-derived CD4 ${ }^{+}$T cells. Error bars represent the means \pm SD; ${ }^{* *} \mathrm{p}<0.001$ compared to MLR-devided cells.

by a factor of $0.59 \pm 0.11(\mathrm{p}<0.001)$ (Fig. 6D and E). However, this decrease was significantly less than that observed in MLRs in the presence of oleate ( $\mathrm{p}=0.003$, unpaired t-test) suggesting that oleate is beneficial for FoxP3 expression. 

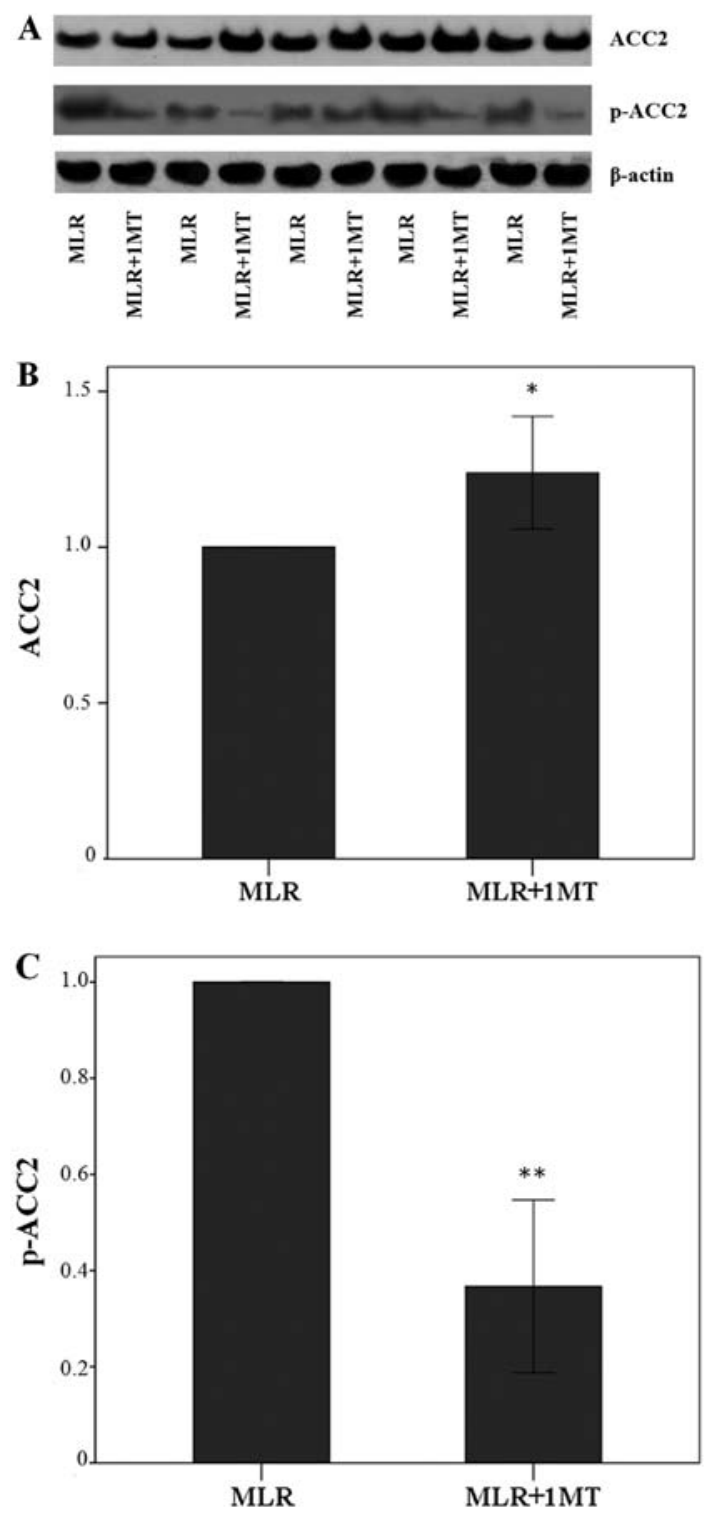

Figure 4. Effect of indoleamine 2,3-dioxygenase (IDO) on ACC2 and p-ACC2 levels in MLR-derived CD4 ${ }^{+}$T-cells. Ten mixed lymphocyte reactions (MLRs) were performed in the presence or absence of the IDO inhibitor 1-DL-methyl-tryptophan (1MT), and CD4 ${ }^{+} \mathrm{T}$-cells were isolated to evaluate the expression of ACC2 and p-ACC2. (A) Western blot analysis results of 5 of the 10 performed experiments are shown. (B) IDO decreased total ACC2 expression, (C) but increased the level of its inactive phosphorylated form $\mathrm{p}-\mathrm{ACC} 2$. Error bars represent the means $\pm \mathrm{SD}$; ${ }^{*} \mathrm{p}<0.05$ and * $\mathrm{p}<0.001$ compared to MLR-devided cells.

In the absence of oleate from the MLRs, 1MT treatment also resulted in an increase in ROR $\gamma \mathrm{t}$ expression in $\mathrm{CD}^{+} \mathrm{T}$-cells by a factor of $2.11 \pm 1.28$ ( $\mathrm{p}<0.001)$ (Fig. $6 \mathrm{D}$ and $\mathrm{F}$ ). This increase did not differ from the increase observed in MLRs performed in the presence of oleate ( $\mathrm{p}=0.772$, unpaired $\mathrm{t}$-test).

\section{Discussion}

Indoleamine 2,3-dioxygenase is expressed in APCs and by degrading L-tryptophan in the microenvironment where the immune response occurs, it suppresses $\mathrm{CD} 4^{+} \mathrm{T}$-cell function by inhibiting cell proliferation, inducing apoptosis and promoting differentiation into Tregs $(20,21)$.
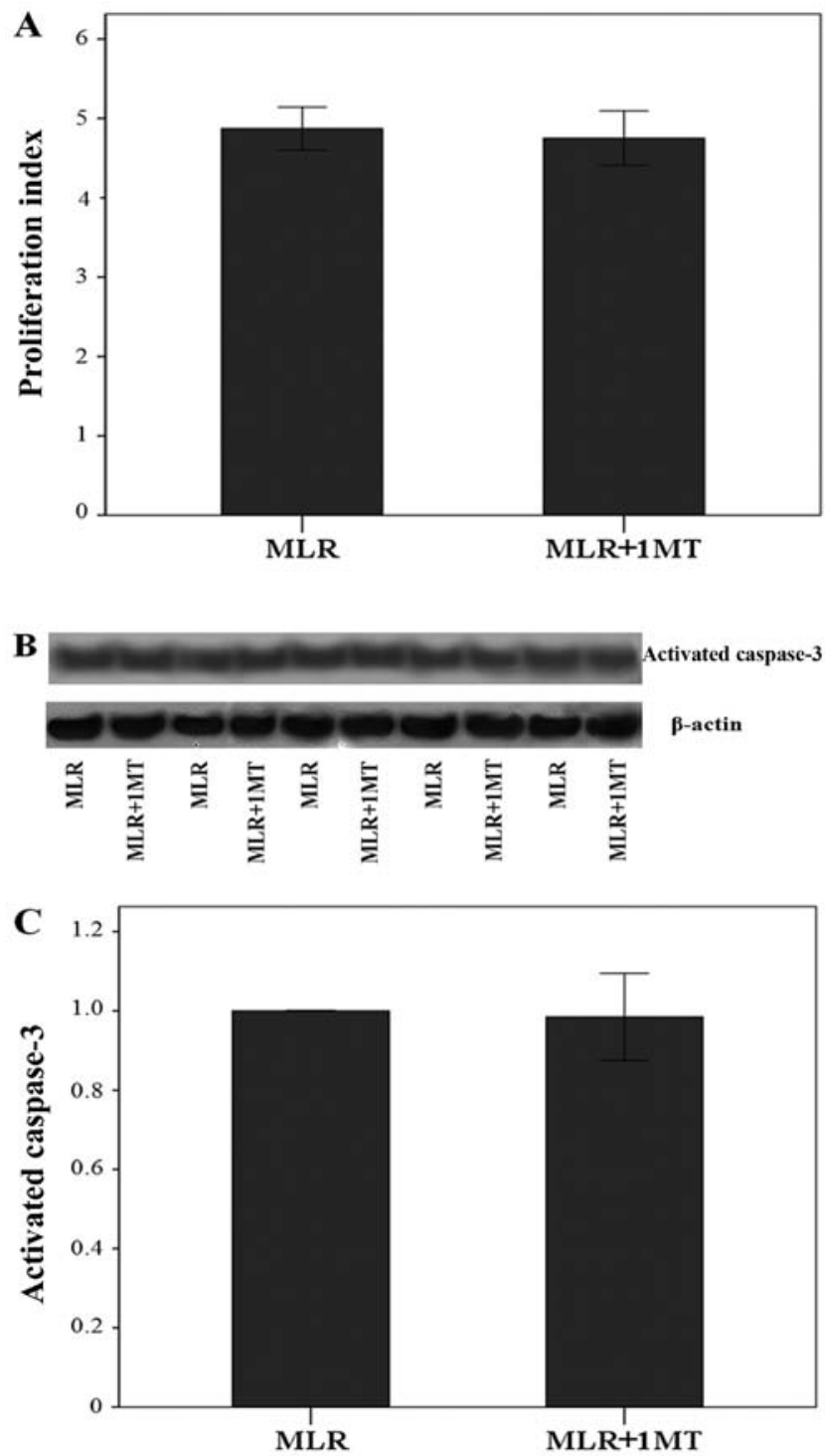

Figure 5. Effect of indoleamine 2,3-dioxygenase (IDO) on cell proliferation in mixed lymphocyte reactions (MLRs) and on activated caspase-3 in MLR-derived CD4 ${ }^{+}$T-cells. Ten MLRs were performed in the presence or not of the IDO inhibitor 1-DL-methyl-tryptophan (1MT). (A) IDO did not affect cell proliferation. Ten MLRs were performed in the presence or absence of the IDO inhibitor $1 \mathrm{MT}$, and $\mathrm{CD}^{+}{ }^{+} \mathrm{T}$ cells were isolated to evaluate the expression of activated caspase-3. (B) Western blot analysis results of 5 of 10 performed experiments are shown. (C) IDO did not affect the level of the activated, terminal in the apoptotic pathway, caspase- 3 . Error bars represent the means \pm SD.

In order to define which of the described pathways are involved in the effect of IDO on CD4 ${ }^{+} \mathrm{T}$ cells (12-14,17-19), a model of alloreactivity, the MLR, was used. In this model, IDO induced L-tryptophan degradation. Decreased L-tryptophan activated the GCN2K pathway since the phosphorylation of its substrate eIF $2 \alpha$ was increased when IDO activity was not blocked by $1 \mathrm{MT}$. This observation is in accordance with previous studies (12-14). Additionally, the present study recapitulates the results of other studies that failed to detect an effect on the other amino-acid sensing system, the mTORC1, since the level of phosphorylation of its substrate, p70S6K, remained unaffected by 1MT (12-14). This is in accordance with findings showing that mTORC1 

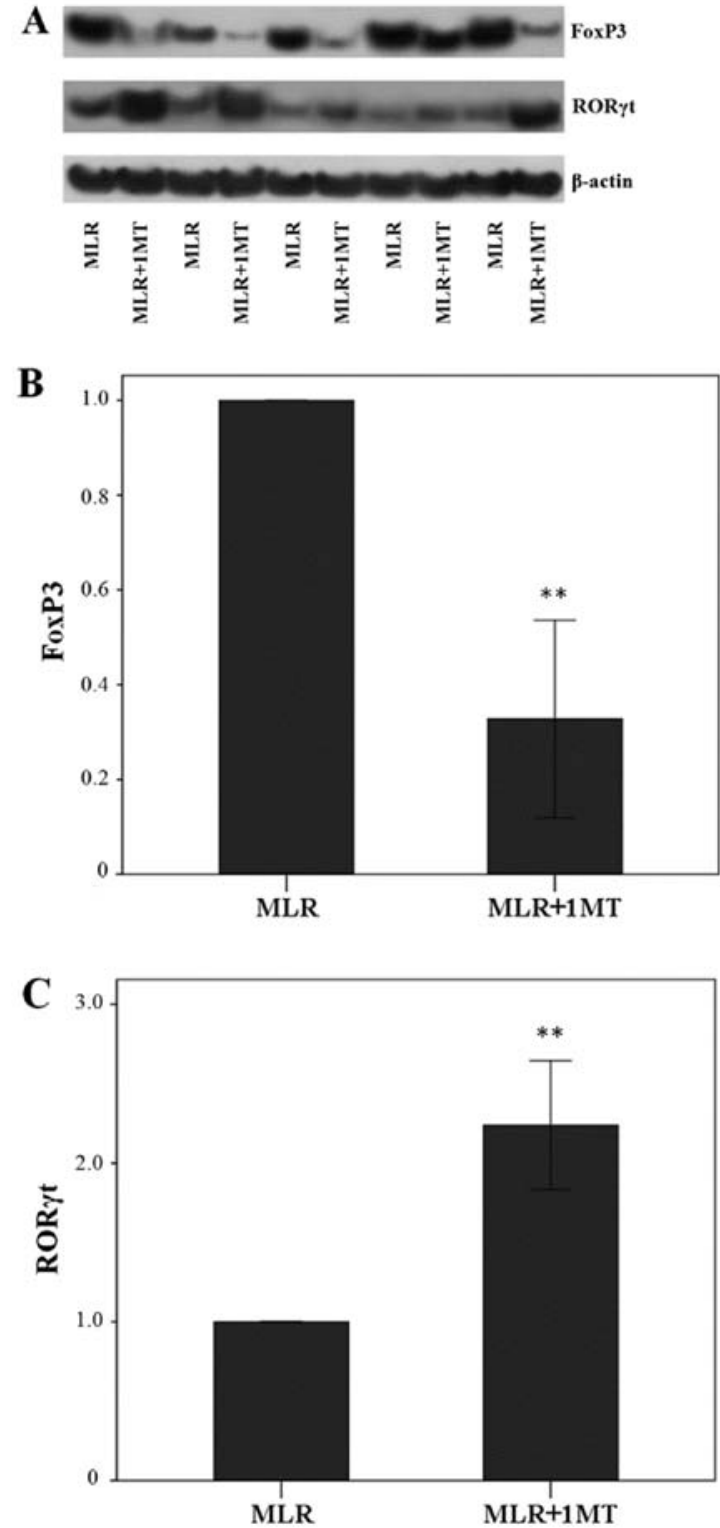

D
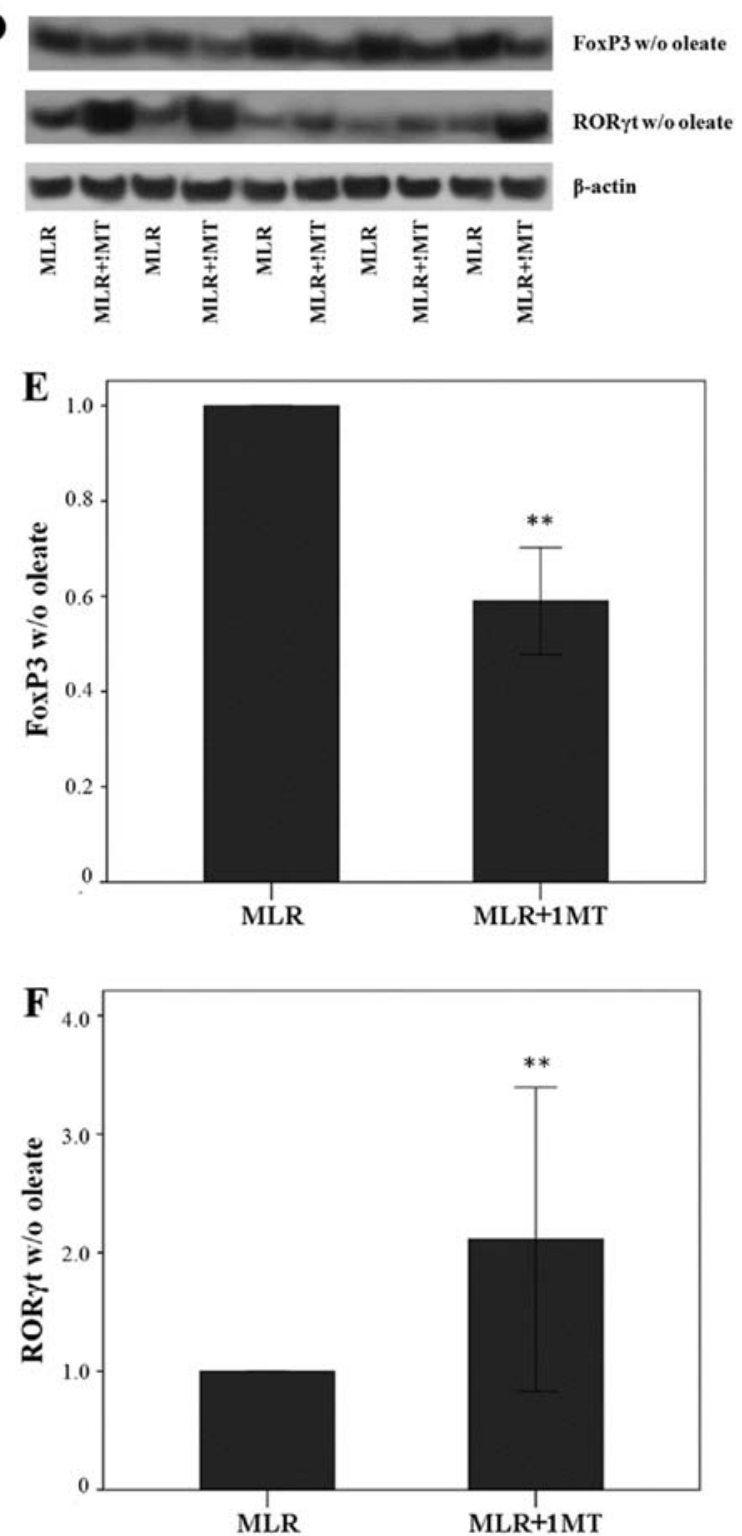

Figure 6. Effect of indoleamine 2,3-dioxygenase (IDO) on forkhead box P3 (FoxP3) and related orphan receptor $\gamma \mathrm{t}$ (ROR $\gamma \mathrm{t}$ ) expression in CD4+ T cells derived from mixed lymphocyte reactions (MLRs) with or without oleate. Ten MLRs were performed in culture media containing or not oleate and in the presence or absence of the IDO inhibitor 1MT. CD4 ${ }^{+} \mathrm{T}$ cells were then isolated to evaluate the expression of FoxP3 and ROR $\gamma \mathrm{t}$. (A) Western blot analysis results of 5 of 10 performed experiments with oleate are shown. (B) IDO increased FoxP3 expression, (C) but decreased ROR $\gamma \mathrm{t}$ expression in MLR-derived CD4+ $\mathrm{T}$ cells. (D) Western blot analysis results of 5 of 10 performed experiments without oleate are shown. In this case, (E) IDO increased FoxP3 expression, (F) whereas it decreased ROR $\gamma \mathrm{t}$ expression in MLR-derived CD4 ${ }^{+} \mathrm{T}$ cells. However, the presence of oleate in the culture medium demonstrated a more profound effect of IDO on FoxP3 expression. Error bars represent the means $\pm \mathrm{SD} ;{ }^{* *} \mathrm{p}<0.001$ compared to MLR-devided cells.

is sensitive to the depletion of certain amino acids; and more precisely of leucine, isoleucine, valine and possibly arginine, but not of tryptophan (31). Furthermore, L-tryptophan depletion and its degradation by IDO results in the production of kynurenine, which may affect $\mathrm{CD}^{+} \mathrm{T}$-cell function $(18,19)$. In the MLR-derived CD $4^{+} \mathrm{T}$ cells, the expression of CYP1A1, a transcriptional target of AhR, was increased in the absence of $1 \mathrm{MT}$, indicating that the IDO kynurenine AhR pathway is associated with our experimental model.

Recent studies have confirmed that IDO may exert its effect on $\mathrm{CD}^{+} \mathrm{T}$ cells by affecting their metabolism (13-16). Specifically, L-tryptophan degradation by IDO has been shown to decrease aerobic glycolysis, glutaminolysis and fatty acid synthesis (13-16), all required for rapid CD4+ ${ }^{+}$-cell proliferation and differentiation towards Teff lineages (22-25). The results of the present study confirmed that L-tryptophan degradation by IDO increased fatty acid consumption in MLRs. In parallel, the activity of CPT1 in MLR-derived CD4 ${ }^{+} \mathrm{T}$ cells increased. Fatty acid $\beta$-oxidation occurs in the mitochondrial matrix. However, acyl-CoAs cannot pass the inner mitochondrial membrane, unless they are converted to acylcarnitine in the cytoplasmic surface of the inner mitochondrial membrane. This reaction is catalyzed by CPT1, which, by controlling the entry of fatty acid into the mitochondrial matrix regulates the rate of fatty acid oxidation $(28,29)$.

The reason for the increased CPT1 activity in $\mathrm{CD}^{+} \mathrm{T}$ cells derived from MLR without the IDO inhibitor, 1MT, may be due to the increased levels of the three CPT1 isoenzymes iden- 
tified in the current study. A possible explanation may depend on the confirmed effect of IDO-induced L-tryptophan degradation in transcription factors such as p53 and cMyc that control cell metabolism in $\mathrm{CD}^{+} \mathrm{T}$ cells $(14,16)$. However, the exact mechanism for this IDO-related increase in CPT1A, CPT1B and CPT1C expression remains to be elucidated.

In addition to CPT1 expression, the activity of this enzyme is tightly regulated and more precisely, is allosterically inhibited by malonyl-CoA. Malonyl-CoA is produced by ACC2, an enzyme associated with the outer mitochondrial membrane $(28,29)$. When IDO activity was not inhibited in MLRs, ACC2 expression in the MLR-derived CD4 ${ }^{+} \mathrm{T}$ cells decreased. In addition, possibly due to AMP-activated protein kinase (AMPK) (32), the phosphorylated inactivated form of ACC2 increased markedly. This IDO-induced alteration in ACC2 is expected to lead to decreased ACC2 activity, decreased malonyl-CoA production and increased CPT1 activity and fatty acid $\beta$-oxidation.

We also evaluated the effect of IDO-induced L-tryptophan degradation on two terminal points of $\mathrm{CD}^{+}{ }^{+} \mathrm{T}$-cell immune response, proliferation and apoptosis. Contrary to what has been shown in a similar experimental model (13-16), IDO did not affect cell proliferation in MLRs, or CD4 ${ }^{+} \mathrm{T}$-cell apoptosis as assessed by activated caspase-3, the terminal caspase at which all the apoptotic pathways converge (33). However, the presence of oleate in the culture medium in the present study yielded different results. Oleate, as a fatty acid, along with the IDO-induced increase in fatty acid oxidation may protect $\mathrm{CD}^{+}{ }^{+} \mathrm{T}$-cells from energy deprivation, since IDO is known to decrease glucose influx in the cell, aerobic glycolysis and glutaminolysis (13-16). The presence of a fatty acid in the culture medium may protect cells from energy deprivation, thus also preventing the inhibition of cell proliferation and the induction of apoptosis. These results also raise the question of whether it is more appropriate to perform immunological experiments using more 'normal' culture medium, which contains fatty acids.

The effect of IDO-induced L-tryptophan degradation on the expression of the Treg signature transcription factor FoxP3, and of the Th17 signature transcription factor, ROR $\gamma \mathrm{t}$, was evaluated (34). The two CD4 ${ }^{+} \mathrm{T}$-cell lineages are formed reciprocally as regards fatty acid metabolism. Fatty acid synthesis favors differentiation into the Th17 lineage, whereas fatty acid oxidation favors differentiation into Tregs $(25,26)$. According to what is generally considered (35-37), IDO increased FoxP3, but decreased ROR $\gamma \mathrm{t}$ expression in MLR-derived CD4 ${ }^{+} \mathrm{T}$-cells. In order to define the effect ofthe presence of fatty acid in the culture medium, we repeated the experiments without oleate. No difference was detected s regards ROR $\gamma \mathrm{t}$; however, the presence of oleate IDO induced a greater increase in FoxP3 expression. The reason remains to be defined, since various aspects regarding the mechanisms that connect fatty acid metabolism with $\mathrm{CD}^{+}{ }^{+} \mathrm{T}$-cell function, such as post-translational protein modification by lipids or the availability of acetyl-CoA for epigenetic modifications, are under investigation (38). Thus, this raises the question of whether a culture medium containing fatty acids more closely mimics the in vivo conditions and may thus be more suitable for lymphocyte culture studies.

In conclusion, the present study demonstrated that IDO, by degrading L-tryptophan, enhanced CPT1 activity and fatty acid oxidation, and exerted fatty acid-dependent effects in human alloreactive $\mathrm{CD} 4^{+} \mathrm{T}$ cells

\section{References}

1. Seo SK, Choi JH, Kim YH, Kang WJ, Park HY, Suh JH, Choi BK, Vinay DS and Kwon BS: 4-1BB-mediated immunotherapy of rheumatoid arthritis. Nat Med 10: 1088-1094, 2004.

2. Gurtner GJ, Newberry RD, Schloemann SR, McDonald KG and Stenson WF: Inhibition of indoleamine 2,3-dioxygenase augments trinitrobenzene sulfonic acid colitis in mice. Gastroenterology 125: 1762-1773, 2003.

3. Kwidzinski E, Bunse J, Aktas O, Richter D, Mutlu L, Zipp F, Nitsch R and Bechmann I: Indolamine 2,3-dioxygenase is expressed in the CNS and down-regulates autoimmune inflammation. FASEB J 19: 1347-1349, 2005.

4. Alexander AM, Crawford M, Bertera S, Rudert WA, Takikawa O, Robbins PD and Trucco M: Indoleamine 2,3-dioxygenase expression in transplanted NOD Islets prolongs graft survival after adoptive transfer of diabetogenic splenocytes. Diabetes 51: 356-365, 2002.

5. Beutelspacher SC, Pillai R, Watson MP, Tan PH, Tsang J, McClure MO, George AJ and Larkin DF: Function of indoleamine 2,3-dioxygenase in corneal allograft rejection and prolongation of allograft survival by over-expression. Eur J Immunol 36: 690-700, 2006.

6. Li Y, Tredget EE, Ghaffari A, Lin X, Kilani RT and Ghahary A: Local expression of indoleamine 2,3-dioxygenase protects engraftment of xenogeneic skin substitute. J Invest Dermatol 126: 128-136, 2006.

7. Munn DH, Zhou M, Attwood JT, Bondarev I, Conway SJ, Marshall B, Brown C and Mellor AL: Prevention of allogeneic fetal rejection by tryptophan catabolism. Science 281: 1191-1193, 1998.

8. Mellor AL, Sivakumar J, Chandler P, Smith K, Molina H, Mao D and Munn DH: Prevention of T cell-driven complement activation and inflammation by tryptophan catabolism during pregnancy. Nat Immunol 2: 64-68, 2001.

9. Munn DH and Mellor AL: Indoleamine 2,3-dioxygenase and tumor-induced tolerance. J Clin Invest 117: 1147-1154, 2007.

10. Eleftheriadis T, Yiannaki E, Antoniadi G, Liakopoulos V, Pissas G, Galaktidou G and Stefanidis I: Plasma indoleamine 2,3-dioxygenase and arginase type I may contribute to decreased blood T-cell count in hemodialysis patients. Ren Fail 34: 1118-1122, 2012.

11. Eleftheriadis T, Liakopoulos V, Antoniadi G, Stefanidis I and Galaktidou G: Indoleamine 2,3-dioxygenase is increased in hemodialysis patients and affects immune response to hepatitis B vaccination. Vaccine 29: 2242-2247, 2011.

12. Munn DH, Sharma MD, Baban B, Harding HP, Zhang Y, Ron D and Mellor AL: GCN2 kinase in T cells mediates proliferative arrest and anergy induction in response to indoleamine 2,3-dioxygenase. Immunity 22: 633-642, 2005.

13. Eleftheriadis T, Pissas G, Antoniadi G, Liakopoulos V and Stefanidis I: Indoleamine 2,3-dioxygenase depletes tryptophan, activates general control non-derepressible 2 kinase and downregulates key enzymes involved in fatty acid synthesis in primary human $\mathrm{CD}^{+} \mathrm{T}$ cells. Immunology 146: 292-300, 2015.

14. Eleftheriadis T, Pissas G, Antoniadi G, Spanoulis A, Liakopoulos V and Stefanidis I: Indoleamine 2,3-dioxygenase increases p53 levels in alloreactive human T cells, and both indoleamine 2,3-dioxygenase and p53 suppress glucose uptake, glycolysis and proliferation. Int Immunol 26: 673-684, 2014.

15. Eleftheriadis T, Pissas G, Yiannaki E, Markala D, Arampatzis S, Antoniadi G, Liakopoulos V and Stefanidis I: Inhibition of indoleamine 2,3-dioxygenase in mixed lymphocyte reaction affects glucose influx and enzymes involved in aerobic glycolysis and glutaminolysis in alloreactive T-cells. Hum Immunol 74: 1501-1509, 2013.

16. Eleftheriadis T, Pissas G, Antoniadi G, Tsogka K, Sounidaki M, Liakopoulos V and Stefanidis I: Indoleamine 2,3 dioxygenase downregulates $\mathrm{T}$ cell receptor complex $\zeta$ chain and $\mathrm{c}$ Myc, and reduces proliferation, lactate dehydrogenase levels and mitochondrial glutaminase in human T cells. Mol Med Rep 13: 925-932, 2016.

17. Cobbold SP, Adams E, Farquhar CA, Nolan KF, Howie D, Lui KO, Fairchild PJ, Mellor AL, Ron D and Waldmann H: Infectious tolerance via the consumption of essential amino acids and mTOR signaling. Proc Natl Acad Sci USA 106: 12055-12060, 2009. 
18. Mezrich JD, Fechner JH, Zhang X, Johnson BP, Burlingham WJ and Bradfield CA: An interaction between kynurenine and the aryl hydrocarbon receptor can generate regulatory $\mathrm{T}$ cells. J Immunol 185: 3190-3198, 2010.

19. Opitz CA, Litzenburger UM, Sahm F, Ott M, Tritschler I, Trump S, Schumacher T, Jestaedt L, Schrenk D, Weller M, et al An endogenous tumour-promoting ligand of the human aryl hydrocarbon receptor. Nature 478: 197-203, 2011.

20. King NJ and Thomas SR: Molecules in focus: Indoleamine 2,3-dioxygenase. Int J Biochem Cell Biol 39: 2167-2172, 2007.

21. Curti A, Trabanelli S, Salvestrini V, Baccarani M and Lemoli RM The role of indoleamine 2,3-dioxygenase in the induction of immune tolerance: Focus on hematology. Blood 113: 2394-2401, 2009.

22. Maciver NJ, Jacobs SR, Wieman HL, Wofford JA, Coloff JL and Rathmell JC: Glucose metabolism in lymphocytes is a regulated process with significant effects on immune cell function and survival. J Leukoc Biol 84: 949-957, 2008.

23. Fox CJ, Hammerman PS and Thompson CB: Fuel feeds function: Energy metabolism and the T-cell response. Nat Rev Immunol 5: 844-852, 2005.

24. Wang R, Dillon CP, Shi LZ, Milasta S, Carter R, Finkelstein D, McCormick LL, Fitzgerald P, Chi H, Munger J, et al: The transcription factor Myc controls metabolic reprogramming upon T lymphocyte activation. Immunity 35: 871-882, 2011.

25. Berod L, Friedrich C, Nandan A, Freitag J, Hagemann S, Harmrolfs K, Sandouk A, Hesse C, Castro CN, Bähre H, et al: De novo fatty acid synthesis controls the fate between regulatory T and T helper 17 cells. Nat Med 20: 1327-1333, 2014.

26. Michalek RD, Gerriets VA, Jacobs SR, Macintyre AN MacIver NJ, Mason EF, Sullivan SA, Nichols AG and Rathmell JC: Cutting edge: Distinct glycolytic and lipid oxidative metabolic programs are essential for effector and regulatory $\mathrm{CD} 4^{+} \mathrm{T}$ cell subsets. J Immunol 186: 3299-3303, 2011.

27. Sato T, Deiwick A, Raddatz G, Koyama K and Schlitt HJ: Interactions of allogeneic human mononuclear cells in the two-way mixed leucocyte culture (MLC): Influence of cell numbers, subpopulations and cyclosporin. Clin Exp Immunol 115 301-308, 1999.
28. Lopaschuk GD, Ussher JR, Folmes CD, Jaswal JS and Stanley WC: Myocardial fatty acid metabolism in health and disease. Physiol Rev 90: 207-258, 2010.

29. Schreurs M, Kuipers F and van der Leij FR: Regulatory enzymes of mitochondrial beta-oxidation as targets for treatment of the metabolic syndrome. Obes Rev 11: 380-388, 2010.

30. Bieber LL and Fiol C: Purification and assay of carnitine acyltransferases. Methods Enzymol 123: 276-284, 1986.

31. Gallinetti J, Harputlugil E and Mitchell JR: Amino acid sensing in dietary-restriction-mediated longevity: Roles of signaltransducing kinases GCN2 and TOR. Biochem J 449: 1-10, 2013.

32. Mihaylova MM and Shaw RJ: The AMPK signalling pathway coordinates cell growth, autophagy and metabolism. Nat Cell Biol 13: 1016-1023, 2011.

33. Fadeel B and Orrenius S: Apoptosis: A basic biological phenomenon with wide-ranging implications in human disease. J Intern Med 258: 479-517, 2005.

34. Raphael I, Nalawade S, Eagar TN and Forsthuber TG: T cell subsets and their signature cytokines in autoimmune and inflammatory diseases. Cytokine 74: 5-17, 2015.

35. Fallarino F, Grohmann U, You S, McGrath BC, Cavener DR, Vacca C, Orabona C, Bianchi R, Belladonna ML, Volpi C, et al: The combined effects of tryptophan starvation and tryptophan catabolites down-regulate $T$ cell receptor zeta-chain and induce a regulatory phenotype in naive T cells. J Immunol 176: 6752-6761, 2006.

36. Sharma MD, Baban B, Chandler P, Hou DY, Singh N, Yagita H, Azuma M, Blazar BR, Mellor AL and Munn DH: Plasmacytoid dendritic cells from mouse tumor-draining lymph nodes directly activate mature Tregs via indoleamine 2,3-dioxygenase. J Clin Invest 117: 2570-2582, 2007.

37. Sharma MD, Hou DY, Liu Y, Koni PA, Metz R, Chandler P, Mellor AL, He Y and Munn DH: Indoleamine 2,3-dioxygenase controls conversion of Foxp3 ${ }^{+}$Tregs to TH17-like cells in tumordraining lymph nodes. Blood 113: 6102-6111, 2009.

38. Lochner M, Berod L and Sparwasser T: Fatty acid metabolism in the regulation of $\mathrm{T}$ cell function. Trends Immunol 36: 81-91, 2015. 